\title{
Impedance Analysis and Optimization of Self-Powered Interface Circuit for Wireless Sensor Nodes Application
}

\author{
Yuan Dong, ${ }^{1}$ Dezhi Li, ${ }^{2}$ Benjamin Ducharne, ${ }^{3}$ Xiaohui Wang, ${ }^{2}$ Jun Gao, ${ }^{2}$ and Bin Zhang $\mathbb{I D}^{2}$ \\ ${ }^{1}$ School of Electronic Control, Chang'an University, Xi'an, China \\ ${ }^{2}$ School of Mechanical, Electrical \& Information Engineering, Shandong University, Weihai, China \\ ${ }^{3}$ Laboratoire de Génie Electrique et Ferroélectricité, INSA de Lyon, Lyon, France \\ Correspondence should be addressed to Bin Zhang; bin.zhang@sdu.edu.cn
}

Received 16 June 2018; Accepted 29 August 2018; Published 24 September 2018

Academic Editor: Md Abdul Halim Miah

Copyright (c) 2018 Yuan Dong et al. This is an open access article distributed under the Creative Commons Attribution License, which permits unrestricted use, distribution, and reproduction in any medium, provided the original work is properly cited.

\begin{abstract}
Energy harvesting for self-powered wireless sensor networks (WSNs) is increasingly needed. In this paper, a self-powered WSN node scenario is proposed and realized by coupling the electric charge extraction interface circuit, power management module, and wireless communication module. Firstly, the output power of an optimized self-powered energy extraction circuit is compared with different energy extraction circuits under various loads and excitation amplitudes theoretically. Then, an energy-harvesting setup is established to validate the load-carrying capacity and working condition of the self-powered optimized synchronized switch harvesting on inductor (SP-OSSHI) circuit. It gives guidance to select and estimate the appropriate energy-consuming level for the sensor and modules. Finally, by connecting the energy-harvesting system, power management element, and sensing part together, a self-powered wireless sensor node is accomplished. Under $18 \mathrm{~Hz}$ resonant excitation, the whole self-powered system transmits 32 bytes of data every 30 seconds including the acceleration and environment temperature. This prototype strongly proves the feasibility of the self-powered WSN node. These research results have potential to be used in different application fields.
\end{abstract}

\section{Introduction}

With the continuous advancement in integrated circuit, microelectronics, low-power sensors, and wireless communications technology, the emergence and development of wireless sensor networks (WSNs) have been given more attention and been widely used $[1,2]$. For example, Sarkar and Misrab [3] proposed that a miniature wireless sensor could be implanted into a human body to detect blood glucose concentration, $\mathrm{pH}$ value, and oxygen content and identify cancer cells, etc. Winkler et al. [4] used wireless sensor networks to disseminate a large number of sensing nodes around important targets such as temporary command posts. By deploying them in advance, once the enemies entered the detection range of the sensing node, the WSNs could detect their position, movement speed, and other information. As an important part of wireless sensor networks, wireless sensor nodes are widely distributed. If batteries are used as the power supply, their lifespan and replacement cost in harsh environments would be a serious problem [5-8]. In addition, in many applications, due to the location of the device (e.g., aerospace), battery replacement is cumbersome or impose unnecessary risks (e.g., implantable biomedical devices such as pacemakers) [9]. Therefore, wireless sensor network nodes need an independent, longlasting, maintenance-free self-powered power supply. Energy-harvesting technology can convert environmental energy (such as light energy, heat energy, mechanical energy, electromagnetic energy, biochemical energy, and wind energy) into electrical energy and provide power for microelectromechanical devices $[10,11]$. Sensors based on MEMS technology consume only microwatts to milliwatts of power to operate properly, so energy-harvesting technology can meet their power needs.

In the industrial production and daily life, vibration is widely distributed, with high energy density and various forms [12]. Especially in implantable structures, vibration energy can be converted into electric energy, providing 
a stable power supply for MEMS devices. Compared with other types of vibration energy-harvesting methods (electromagnetic [13] and electrostatic [14]), piezoelectric vibration energy harvesting has significant advantages in terms of structural size, material properties, output power, and stability and can be an ideal power supply solution for MEMS devices [15].

Between the piezoceramic and load, an interface is needed to extract as much electricity as possible from the piezoceramic and rectify the unstable AC current. The most basic energy-harvesting circuit is called the standard energyharvesting (SEH) circuit, composed of a rectifier bridge, a filter capacitor, and a load resistor. Guyomar et al. [16] and Lefeuvre et al. [17] proposed a nonlinear technique named "synchronized switch harvesting on inductor (SSHI)" and "synchronous electric charge extraction (SECE)," significantly increasing the output power. Under the same displacement excitation, SSHI can increase the harvested power by 9 times, compared to SEH. Nonlinear techniques applied to the resonant mode or pulse mode make the harvesting device smaller and the coupling coefficient lower. The SECE technology combines the nonlinear technology with a DCDC converter structure to enable energy extraction 4 times higher than the SEH technology, and energy extraction is independent of load resistance or output voltage [18]. Lallart et al. $[19,20]$ proposed the double synchronized switch harvesting (DSSH), which consists of two parts: the series inductor and the buck-boost converter. After theoretical analysis and experimental verification, the output power of the circuit is independent of the load and the power is increased by 5 times, compared to SEH circuit. Wu et al. [21] simplified the optimized synchronous electric charge extraction (OSECE) and replaced the rectifier bridge with a flyback transformer with two primary windings connected to the piezoceramic. The circuit retains the advantages of the SECE circuit and further improves the extraction efficiency. Zhang et al. $[22,23]$ proposed an Ericsson energy extraction technology for bulk piezoelectric ceramics which can significantly increase the energy output power and applied it to ocean wave power generation. Lefeuvre et al. [24] proposed phase-shifted synchronous electric charge extraction (PS-SECE) to control the phase-shift phase angle using the maximum power point tracking algorithm. Under the electromechanical coupling conditions, the energy extraction efficiency can be significantly improved, and the frequency bandwidth is greatly increased. Under high electromechanical coupling conditions, the maximum power of the PS-SECE circuit can be about twice that of a conventional SECE circuit. In the self-powered system, the acquisition of energy is critical. Some researchers theoretically study the efficiency of energy collection under different excitations [2], improve the electromechanical coupling [25], and examine how to collect energy with wider frequency and wider vibration [26]. Zhang et al. [27] proposed a vehicle-bridge coupling platform to study and experiment the energy extraction of piezoelectric ceramics under different positions and vehicle conditions, which provided a theoretical reference for the layout of the energy extraction module on the bridge health-monitoring sensor network.
Su et al. [28] proposed a set of steel corrosion-monitoring devices that use piezoelectric energy harvesting to provide self-power supply, which can automatically predict the corrosion process and take appropriate mitigation measures based on the data information to extend the service life and improve the building safety factor. Through the above analysis and conclusion, we can see that the high-efficiency piezoelectric energy-harvesting circuit can fully extract the electrical energy generated by the piezoelectric ceramic and greatly increase the energy output power [29]. Therefore, the piezoelectric energy-harvesting technology has great application prospects in self-powered devices such as wireless sensing, structural health monitoring, and corrosion damage prediction.

In order to make the self-powered circuit easy to build and easy to integrate with the sensor and comprehensively consider the energy extraction capability, we chose the selfpowered optimized synchronized switch harvesting on inductor (SP-OSSHI) for the wireless transmission module. The SP-OSSHI circuit uses a multiplex method of rectifier circuit and peak detection module. It has the ability to rectify while also enabling charge extraction. In this study, its carrying capacity under various load conditions has been studied and compared with other interface circuits. At the same time, the selection of power management modules for the extracted electrical energy is particularly important. The power management module optimizes system-operating time, enhances system stability, and optimizes the energy configuration [30]. Based on the above considerations, the use of LTC3588 as a module for power management at the nanowatt level is fully capable. The communication module is a commercial product from TI. Modeling simulation and experiment have been conducted to validate the feasibility of this project.

\section{Electromechanical Coupling Model and Interface Circuit Analysis}

2.1. Piezoceramic Electromechanical Coupling Model. The piezoelectric energy-harvesting devices make use of positive piezoelectric effect to convert mechanical vibration of the base structure into electrical energy. Because of the external stress applied to a piezoceramic, the wall movement induces the electric charges accumulated on both sides of the electrodes.

The establishment of the electromechanical coupling model is to combine the electric factor with the mechanical factor, as shown in Figure 1. The mechanical loss is equivalent to the viscous damper $C, M$ is the mass, $K_{\mathrm{S}}$ is the equivalent stiffness of the structure, $F$ is the external excitation, and $u$ is the structural displacement.

\subsection{Interface Circuit Analysis}

2.2.1. Standard Energy-Harvesting Circuit. The standard energy-harvesting (SEH) circuit is composed of a full-bridge rectifier and a filter capacitor $C_{\mathrm{REC}}$ in parallel. The full-bridge rectifier converts the alternating current generated by the 


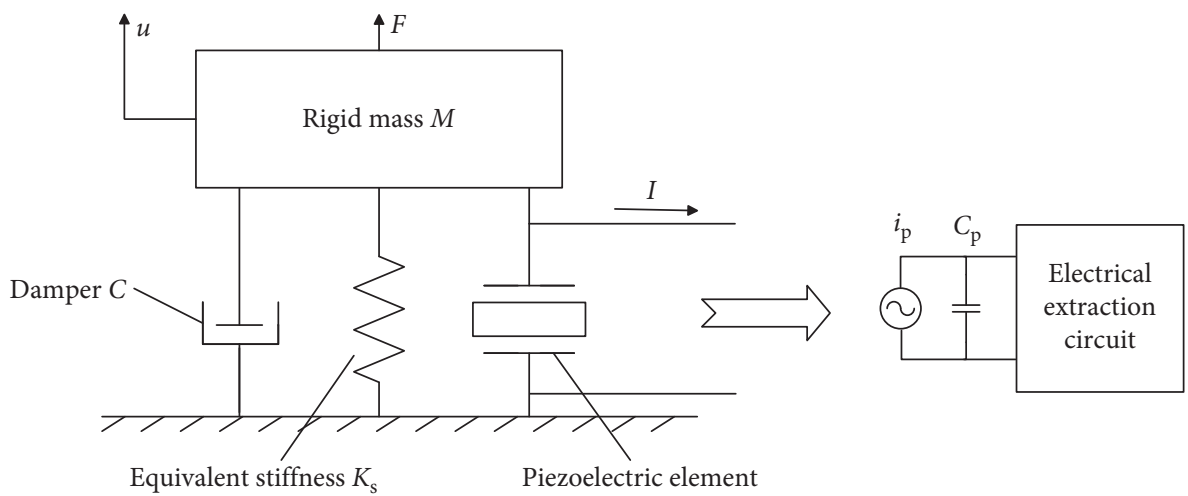

FIGURE 1: Electromechanical coupling model.

piezoceramic into a direct current. The filter capacitor $C_{\mathrm{REC}}$ outputs a constant DC supply for the load $R$, as shown in Figure 2.

The rectifier bridge works only if the absolute value, $V_{\mathrm{P}}$ (can be expressed by Equation (1)), on the piezoceramic equivalent capacitor, $C_{\mathrm{P}}$, exceeds $V_{\mathrm{DC}}$ plus the diode threshold. The electric charge will transfer from $C_{\mathrm{P}}$ to the rectifying capacitor, $C_{\mathrm{REC}}$. The output voltage $V_{\mathrm{DC}}$ and output power of the SEH circuit $P_{\mathrm{SEH}}$ can be expressed as follows:

$$
\begin{aligned}
\int V_{\mathrm{P}} I d t & =\int \alpha V_{\mathrm{P}} \dot{u} d t-\frac{1}{2} C_{\mathrm{P}} V_{\mathrm{P}}^{2}, \\
V_{\mathrm{DC}} & =\frac{\omega \alpha U_{\mathrm{M}} R}{\omega R C_{\mathrm{P}}+(\pi / 2)}, \\
P_{\mathrm{SEH}} & =\frac{V_{\mathrm{DC}}^{2}}{R}=\frac{\omega^{2} \alpha^{2} U_{\mathrm{M}}^{2} R}{\left(\omega R C_{\mathrm{P}}+(\pi / 2)\right)^{2}},
\end{aligned}
$$

where $\left(C_{\mathrm{P}} V_{\mathrm{P}}^{2}\right) / 2$ is the energy stored in the equivalent capacitor; thus, $\int V_{\mathrm{P}} I d t$ is the energy actually flowing into the subsequent collection circuit and used for the load. $\alpha$ is the force factor of the piezoceramic, $\omega$ is the vibrational angular velocity of the system, and $U_{\mathrm{M}}$ is the vibration displacement amplitude of the piezoceramic. The optimal load $R_{\text {opt }}$ of the SEH circuit can be obtained from Equation (3):

$$
\frac{\partial P_{\mathrm{SEH}}}{\partial R}=0 \Longrightarrow R_{\mathrm{opt}}=\frac{\pi}{2 \omega C_{\mathrm{P}}} .
$$

Equations (3) and (4) lead to the maximum output power $\left(P_{\mathrm{SEH}}\right)_{\max }$ of the SEH circuit:

$$
\left(P_{\mathrm{SEH}}\right)_{\max }=\frac{\omega \alpha^{2} U_{\mathrm{M}}^{2}}{2 \pi C_{\mathrm{P}}} \text {. }
$$

2.2.2. Series Synchronized Switch Harvesting on Inductor Circuit. Series synchronized switch harvesting on inductor (S-SSHI) connects an inductor $L$ and a switch $S$ in series between the piezoceramic and the full-bridge rectifier, as shown in Figure 3.

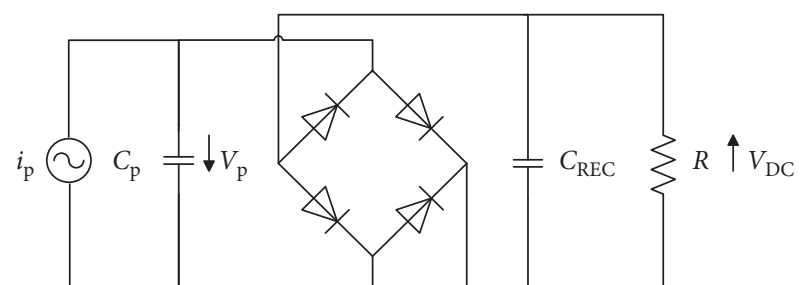

FIgURE 2: Schematic of the standard energy extraction circuit.

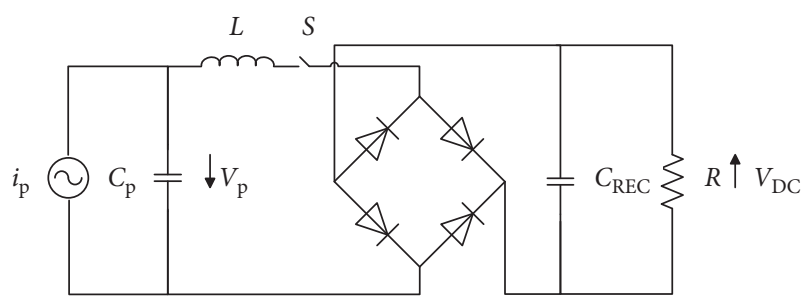

FIGURE 3: Schematic of the series synchronized switch harvesting on inductor circuit.

The working principle of the circuit is to close the switch $S$ at the amplitude of the mechanical vibration. At these times, a high-frequency oscillating electrical circuit $L-C_{\mathrm{P}}$ is established. Its oscillation period $\tau$ is much smaller than the mechanical vibration period $T$. When the oscillating circuit is working for half a cycle, the polarity of the voltage across the piezoceramic will instantly reverse, and the voltage is reversed from $V_{\mathrm{M}}$ to $V_{\mathrm{m}}$. Losses occur during flipping. Most of the losses are caused by the inductor. The quality factor introduced into the inductor is $Q_{L}$. The alternating current amplitude of the inductor is inversely proportional to the power supply frequency. The synthetic effect of inductance and frequency is called inductive reactance, and the expression is shown in Equation (6), where $X_{\mathrm{L}}$ is the inductive reactance, $f$ is the frequency, and $L$ is the inductance. $Q_{\mathrm{L}}$ is the ratio of stored energy to energy consumed by the energy storage component, which can be abbreviated as $Q_{\mathrm{L}}=X_{\mathrm{L}} / R_{\mathrm{DC}}$, where $R_{\mathrm{DC}}$ is the series resistance. The flipping factor is $m$. The relationship between the absolute value of the voltage across the piezoelectric ceramic $V_{\mathrm{DC}}$ and the absolute value of the reversed voltage $V_{\mathrm{m}}$ is expressed as Equation (7): 


$$
\begin{aligned}
X_{\mathrm{L}} & =2 \pi f L, \\
V_{\mathrm{m}}-V_{\mathrm{DC}} & =e^{-\left(\pi /\left(2 \mathrm{Q}_{\mathrm{L}}\right)\right)}\left(V_{\mathrm{M}}-V_{\mathrm{DC}}\right)=m \cdot\left(V_{\mathrm{M}}-V_{\mathrm{DC}}\right),
\end{aligned}
$$

so $m=e^{-\left(\pi /\left(2 Q_{\mathrm{L}}\right)\right)}$.

The output voltage $V_{\text {DC }}$ and output power of S-SSHI $P_{\text {S-SSHI }}$ can be expressed as follows:

$$
\begin{aligned}
V_{\mathrm{DC}} & =\frac{\omega \alpha U_{\mathrm{M}} R(1+m)}{\omega R C_{\mathrm{P}}(1+m)+((\pi / 2)(1-m))}, \\
P_{\mathrm{S}-\mathrm{SSHI}} & =\frac{V_{\mathrm{DC}}^{2}}{R}=\frac{\omega^{2} \alpha^{2} U_{\mathrm{M}}^{2} R(1+m)^{2}}{\left[\omega R C_{\mathrm{P}}(1+m)+((\pi / 2)(1-m))\right]^{2}} .
\end{aligned}
$$

The optimal load $R_{\text {opt }}$ of the S-SSHI circuit can be obtained from Equation (9):

$$
\frac{\partial P_{\mathrm{S}-\mathrm{SSHI}}}{\partial R}=0 \Longrightarrow R_{\mathrm{opt}}=\frac{\pi}{2 \omega C_{\mathrm{P}}} \cdot \frac{1-m}{1+m} .
$$
is

The corresponding maximum output power $\left(P_{\mathrm{S}-\mathrm{SSHI}}\right)_{\max }$

$$
\left(P_{\mathrm{S}-\mathrm{SSHI}}\right)_{\max }=\frac{\omega \alpha^{2} U_{\mathrm{M}}^{2}}{2 \pi C_{\mathrm{P}}} \cdot \frac{1+m}{1-m} .
$$

By comparing Equations (5) and (11), Equation (12) can be obtained:

$$
\frac{\left(P_{\mathrm{S}-\mathrm{SSHI}}\right)_{\max }}{\left(P_{\mathrm{SEH}}\right)_{\max }}=\frac{1+m}{1-m}
$$

From Equation (12), when the flipping factor is usually between 0.5 and 0.8 , the maximum output power of S-SSHI is 3-9 times, compared to SEH. However, in the S-SSHI technique, the switch is generally controlled by an external synchronized square wave, which consumes extra power and needs a peak detection technique. To further simplify the auxiliary devices, a self-powered peak detection circuit is introduced by Lallart and Guyomar [31], Richard et al. [32], and Liang and Liao [33].

\subsubsection{Self-Powered Optimized Synchronized Switch Har-} vesting on Inductor Circuit. The self-powered optimized synchronized switch harvesting on inductor (SP-OSSHI) circuit used in this paper is shown in Figure 4. An improvement was made based on the S-SSHI, replacing the switch with two passive peak detection modules, as shown in Figure 5. To realize the on-off of the switch at the maximum and minimum values of the vibration displacement, the peak detection module is composed of an envelope detector, a comparator, and a switch.

The working principle of the passive peak detection module is as follows: resistor $R_{1}$, diode $D_{1}$, and capacitor $C_{1}$ form an envelope detector to detect the voltage signal of the piezoceramic. When the piezoceramic voltage rises, it charges the capacitor $C_{1}$. At this time, the transistors $T_{1}$ and $T_{2}$ are blocked; $R_{\mathrm{M}}$ will ensure that the base-emitter voltages of $T_{1}$ and $T_{2}$ do not interfere with the envelope detector and capacitor $C_{1}$; when the difference between the input voltage and the voltage of the capacitor $C_{1}$ is larger than the threshold voltage of $T_{1}$, the transistor $T_{1}$ turns on. The capacitor $C_{1}$ discharges through $T_{1}, D_{2}$, and $T_{2}$. Therefore, $T_{2}$ turns on. The capacitor $C_{\mathrm{P}}$ discharges through $D_{3}$ and $T_{2}$, which is the energy extraction process.

In the positive half cycle of the piezoceramic output voltage signal, the circuit can be divided into the following four working phases.

(1) Stage I: Circuit Charge Stage. The voltage of the piezoceramic increases as the positive displacement increases. The capacitors $C_{\mathrm{P}}, C_{1}$, and $C_{2}$ begin to charge. The voltage across the capacitor increases, and all transistors in the circuit are blocked. When the displacement of the piezoceramic reaches a maximum value, the voltage across the capacitors $C_{\mathrm{P}}, C_{1}$, and $C_{2}$ also reaches a maximum value. The current flows as follows: $\mathrm{B} \longrightarrow i_{\mathrm{p}} \longrightarrow \mathrm{A}$ and $\mathrm{A} \longrightarrow$ $C_{\mathrm{P}}\left\|R_{1} D_{1} C_{1}\right\| R_{\mathrm{N}} T_{4} C_{2} \longrightarrow \mathrm{B}$.

(2) Stage II: Voltage Reversal Stage. As the displacement of the piezoceramic starts to reverse, the voltage on the capacitor $C_{\mathrm{P}}$ decreases. When the difference between the input voltage and the voltage of the capacitor $C_{1}$ is greater than the threshold voltage of $T_{1}$, the transistor $T_{1}$ turns on. The capacitor $C_{1}$ discharges through $T_{1}, R_{\mathrm{M}}, D_{2}, T_{2}$, $C_{\text {REC }}, D_{8}$, and $L$. Therefore, $T_{2}$ conducts. $C_{\mathrm{P}}$ and $L$ constitute an oscillation circuit through $D_{3}, T_{2}$, $C_{\text {REC }}$, and $D_{8}$ so that the voltage across the piezoceramic is rapidly reversed. The current flows as follows: $\mathrm{B} \longrightarrow \mathrm{C}_{\mathrm{P}} \longrightarrow \mathrm{A} \longrightarrow\left(\mathrm{B} \longrightarrow \mathrm{C}_{1} \longrightarrow T_{1} \longrightarrow\right.$ $\left.D_{2}\right) R_{\mathrm{M}} T_{1} D_{2}\left\|\quad D_{3} \longrightarrow T_{2} \longrightarrow C_{\mathrm{REC}}\right\| R \longrightarrow D_{8} \longrightarrow L$ $\longrightarrow \mathrm{B}$ and $\mathrm{B} \longrightarrow \mathrm{C}_{1} \longrightarrow T_{1}$.

(3) Stage III: Inductor Freewheeling Stage. After the voltage reversal across the piezoceramic is completed, the transistor $T_{2}$ is blocked. Inductor $L$ transfers energy to capacitors $C_{\mathrm{REC}}, C_{1}$, and $C_{\mathrm{P}}$ through diodes and transistor $T_{3}$ to supplement the capacitive dissipation voltage. The current flows as follows: $\mathrm{A} \longrightarrow C_{\mathrm{P}} \| R_{1} D_{1} C_{1} \longrightarrow \mathrm{B} \longrightarrow L D_{7} \longrightarrow$ $C_{\mathrm{REC}} \| R \longrightarrow \mathrm{T}_{3} \mathrm{D}_{6} \longrightarrow \mathrm{A}$.

(4) Stage IV: Charge Neutralization Stage. When the charge in the inductor is completely transferred to the capacitor, the transistors $T_{2}$ and $T_{3}$ are blocked. Capacitor $C_{2}$ has not discharged yet, and the extra charge in $C_{2}$ will flow into $C_{\mathrm{P}}$ and $C_{1}$ until the three voltages are the same. The current flows as follows: $\mathrm{A} \longrightarrow C_{\mathrm{P}} \| R_{1} D_{1} C_{1} \longrightarrow \mathrm{B} \longrightarrow C_{2} D_{4} R_{2} \longrightarrow \mathrm{A}$. Positive half-cycle energy extraction is completed. Negative half-cycle energy extraction is performed in a similar way.

\section{Simulation Analysis}

The proposed SP-OSSHI circuit is modeled and simulated in Simulink, and the circuit simulation waveforms are obtained. Figure 6 is the simulation waveform of the output 


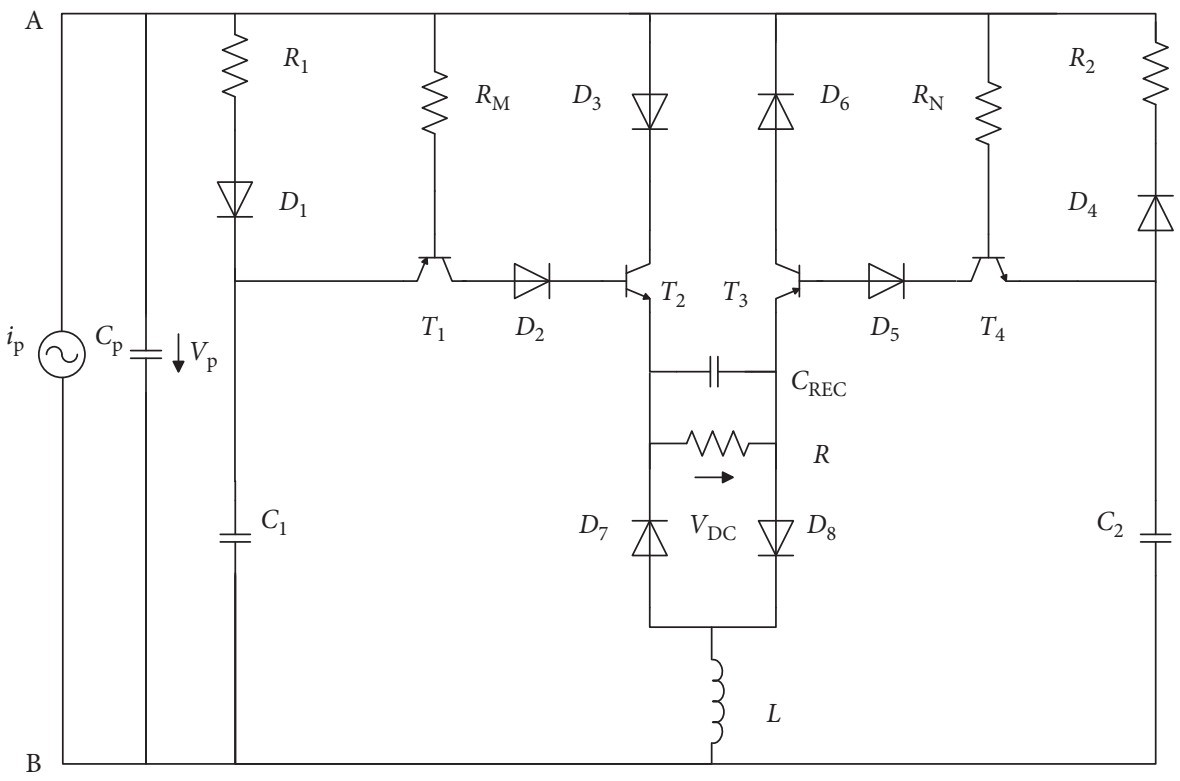

FIGURE 4: Schematic of the self-powered optimized synchronized switch harvesting on inductor circuit.

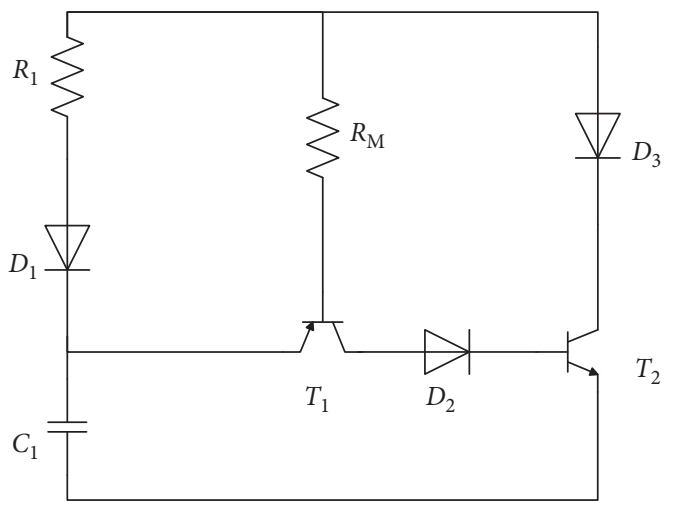

Figure 5: Passive peak detection module.

voltage $V_{\mathrm{p}}$. Figure 7 is the current waveform of the inductor $L$. The first flip corresponds to Stage II (voltage reversal stage) in the SP-OSSHI circuit, and the second flip corresponds to Stage III (inductor freewheeling stage). As shown in Figure 6, the output voltage $V_{\mathrm{P}}$ of the piezoceramic is reversed after the peak. This is because the voltage drop of the threshold voltage of the diode and the transistor in the passive peak detection module leads to a certain phase lag between the system peak voltage and the actual switching time. After the reverse phase of the voltage, the vibration displacement continues to move to the negative direction, and the voltage $V_{\mathrm{P}}$ of the piezoceramic also drops to the reverse peak. The peak detection module works again, and the similar process is repeated. From the above process, it is easy to see that the switch conducts two times in each vibration cycle. The switch conducts at the positive and negative values of the vibration displacement, respectively. At this point, the inductor $L$ and capacitor $C_{\mathrm{P}}$ constitute $L-C_{\mathrm{P}}$ oscillating circuit. After one-fourth of the oscillation period, the energy in the capacitor $C_{\mathrm{P}}$ is completely transferred to the inductor $L$. As

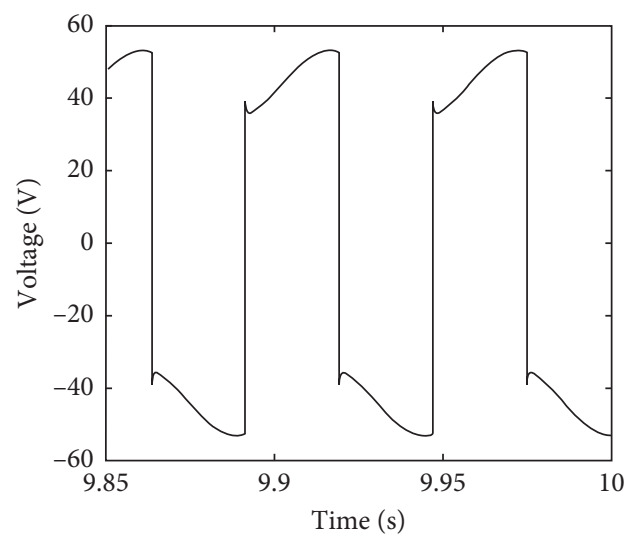

FIGURE 6: Output voltage waveform of a piezoceramic using the SPOSSHI circuit.

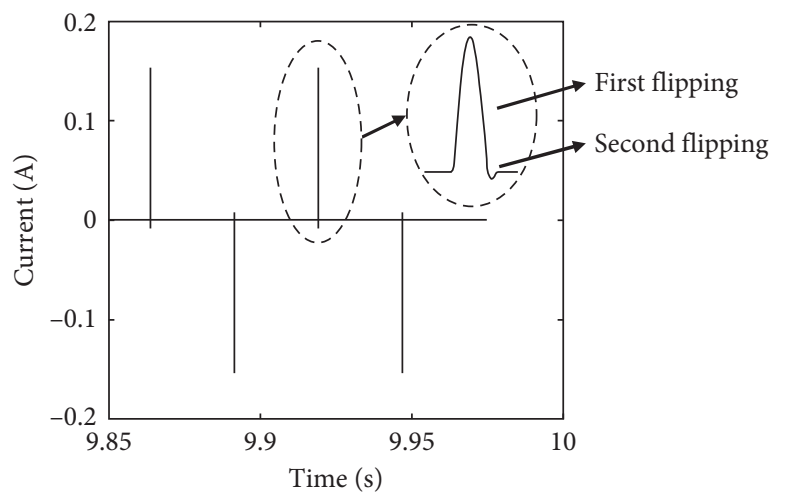

FIGURE 7: Inductor current waveform with the SP-OSSHI circuit.

shown in Figure 7, the current in the inductor $L$ rapidly increases from zero to the maximum and decreases to zero, that is, the first flipping; then the inductor $L$ will continue to discharge current, that is, the second flipping. 
In this paper, the inductor characteristics have been analyzed in detail. The actual inductor can be simulated as 4 passive ideal components: series inductor $(L)$, series resistor $\left(R_{\mathrm{DC}}\right)$, shunt capacitor $\left(C_{\mathrm{L}}\right)$, and parallel resistor $\left(R_{\mathrm{L}}\right)$. The equivalent model of the inductor is shown in Figure 8. $R_{\mathrm{DC}}$ is a DC resistance, which is the resistance measured when the DC current is applied to the inductor. $R_{\mathrm{L}}$ is the loss of the magnetic core. $C_{\mathrm{L}}$ is the distributed capacitance between the inductor inner coil and the lead terminal. To simulate the power output of the circuit under different inductor values, $R_{\mathrm{L}}$ is ignored during simulation. $C_{\mathrm{L}}$ is set to $1 \mathrm{nF}$ by default, $R_{\mathrm{DC}}=2 \pi f L$, and the load resistance is $R=50 \mathrm{k} \Omega$. The relationship between the inductor parameters and the power output is shown in Figure 9. The optimal inductor value appears at $20-24 \mathrm{mH}$.

To illustrate the performance of different interface circuits regarding energy extraction efficiency, the output power of the three circuits under different loads is compared, as shown in Figure 10. The simulation results show that the output power and output efficiency of the SSHI circuit are much better than those of the SEH circuit under the condition of small load $(10 \mathrm{k} \Omega-200 \mathrm{k} \Omega)$. As the S-SSHI circuit switch is controlled by an external control circuit, whose switch conduction time is controllable, its energy extraction efficiency (excluding the controlled power) is better than that of the SP-OSSHI circuit. Since the passive peak detection module exists in the SP-OSSHI circuit, the charge accumulated in the detection capacitor in the detection circuit needs to be discharged through the resistor, which not only is difficult to control but also causes energy loss. Therefore, the SP-OSSHI circuit is lower in power than the S-SSHI circuit. By comparing the maximum output power of the SP-OSSHI circuit and the SEH circuit, the result shows that the output power of the SP-OSSHI circuit is about 5.8 times that of the SEH circuit.

It is noted that the S-SSHI circuit and the SP-OSSHI circuit almost have the same energy extraction efficiency in the ideal condition, while the in-site application of both of them will be weakened by the electric loss, peak detection, and switch control.

\section{Experimental Verification}

4.1. Energy Extraction Circuit Verification. In order to verify the above theoretical and simulation analysis, the experimental setup of the SP-OSSHI circuit proposed above has been performed, as shown in Figure 11.

Experimental equipment mainly includes the piezoceramic, cantilever beam, noncontact vibrator, laser displacement sensor, SP-OSSHI interface circuit, SEH interface circuit, vibration console, and oscilloscope.

The cantilever beam system is shown in Figure 11. A $50 \mathrm{~mm} \times 50 \mathrm{~mm} \times 0.15 \mathrm{~mm}$ piezoceramic is attached to the fixed end of the cantilever beam. A noncontact vibrator is arranged above the free end, which is controlled by the vibration console in different frequencies and excitation amplitude. An oscilloscope (ROHDE\&SCHWARZ, RTB2004) was used to measure the input and output. The first-order natural frequency of the system was $18 \mathrm{~Hz}$, and

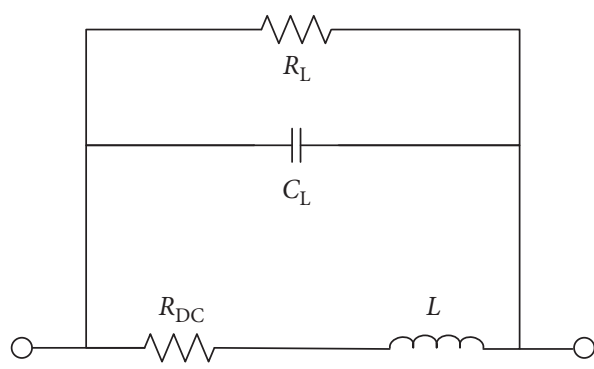

Figure 8: Equivalent model of the inductor.

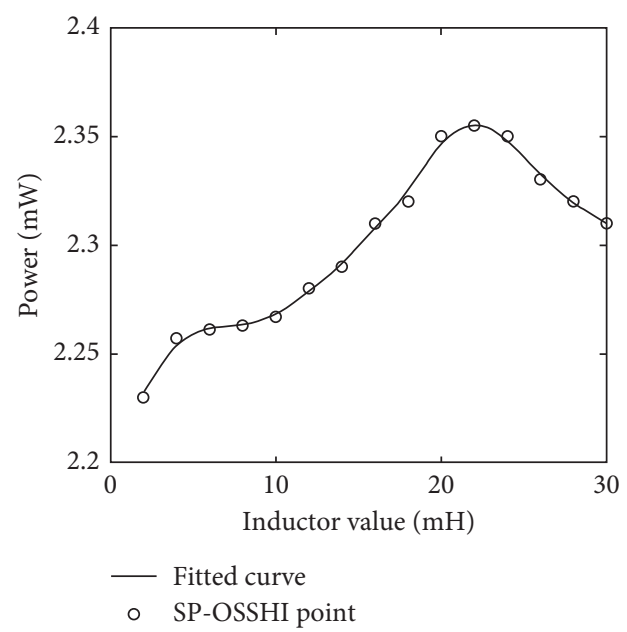

FIGURE 9: Inductor-power simulation waveform.

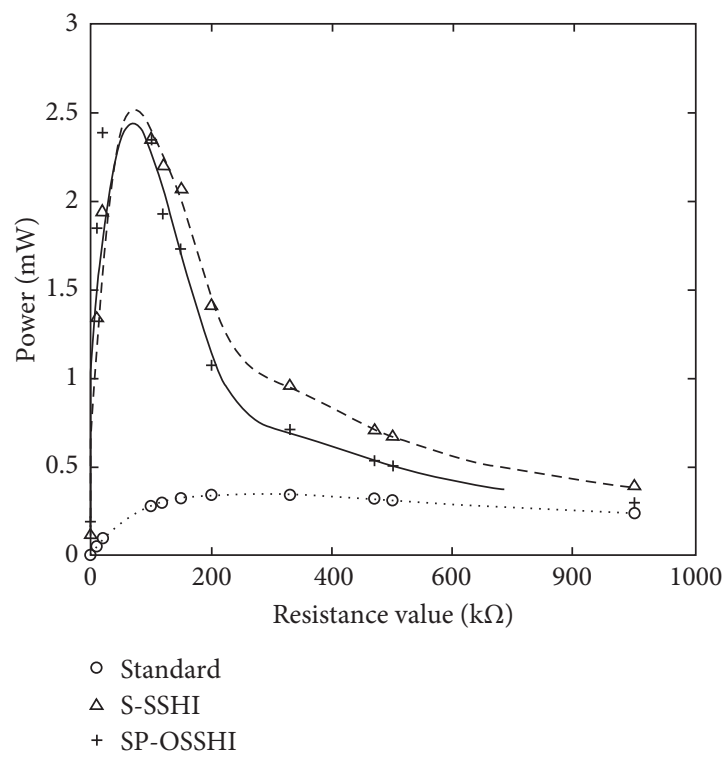

FIgUre 10: Three PEH circuits' output power with different load resistance values.

this was used as the excitation frequency. The component parameters used in the SP-OSSHI interface circuit are shown in Table 1.

The load voltage and responding power output under different load conditions of the SP-OSSHI circuit and the SEH 


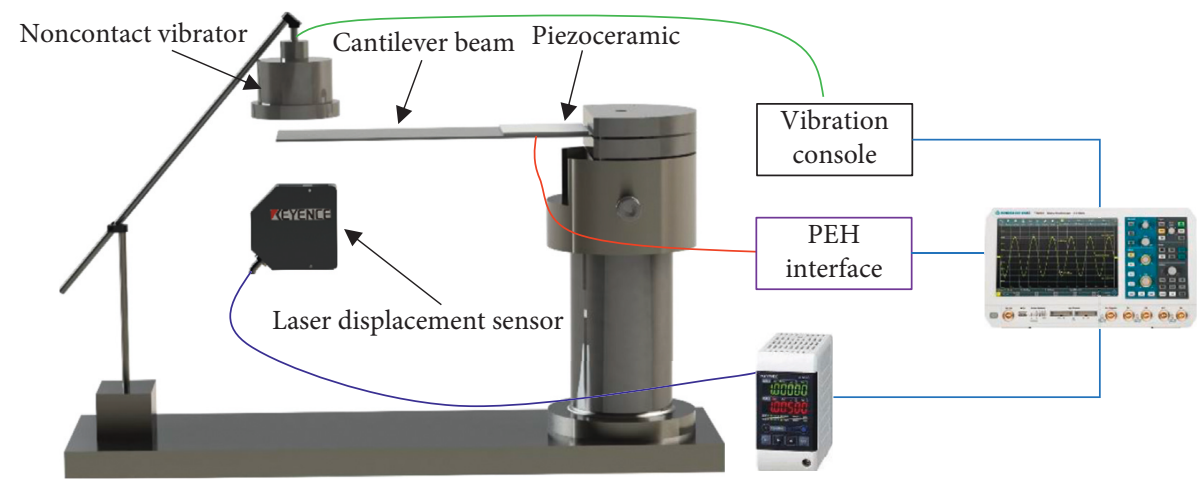

FIGURE 11: Vibration energy-harvesting experimental setup.

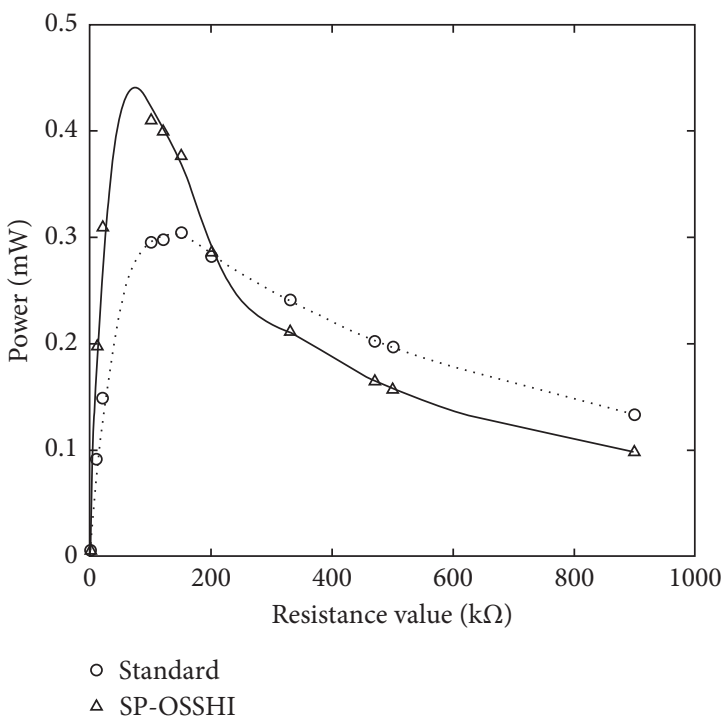

Figure 12: Two PEH circuits' experiment output power with different load resistance values.

TABle 1: Component parameters.

\begin{tabular}{lc}
\hline Components & Parameters \\
\hline$i_{\mathrm{P}}$ & $120 \mu \mathrm{A} / 18 \mathrm{~Hz}$ \\
$C_{\mathrm{P}}$ & $54.1 \mathrm{nF}$ \\
$R_{1}, R_{2}$ & $200 \mathrm{k} \Omega$ \\
Diodes & $1 \mathrm{~N} 4004$ \\
$T_{1}, T_{3}$ & TIP32C \\
$T_{2}, T_{4}$ & TIP31C \\
$L$ & $20 \mathrm{mH}$ \\
$C_{\mathrm{REC}}$ & $10 \mu \mathrm{F}$ \\
$C_{1}, C_{2}$ & $2 \mathrm{nF}$ \\
$R_{\mathrm{M}}, R_{\mathrm{N}}$ & $50 \mathrm{k} \Omega$ \\
\hline
\end{tabular}

circuit are shown in Figure 12. The experimental verification results show that the simulation and experiment of the output power versus different loads coincide with each other. Mainly due to the voltage drop of the diode and transistor threshold, the energy loss caused by the internal resistance of each component and the quality factor of the inductor leads to the eddy current loss of the inductor itself in the actual circuit. In the low-load region, the output voltage of the piezoceramic is low, and the loss comes from the conduction voltage drop of the diode and the thermal energy loss of each resistive element. In the high-load region, the output voltage of the piezoceramic is high, and the energy loss caused by diode and transistor threshold voltage drops is relatively in low percentage, but the energy extraction circuit increases the current in the charge-discharge circuit. The thermal energy loss of the entire circuit resistive element including the internal resistance of the inductor is increased. Comparing the optimal power of the experimental circuit, the output power of the SPOSSHI circuit is about 1.5 times that of the SEH circuit.

The excitation amplitude varies and is validated by the laser displacement sensor (KEYENCE; LK-G3000 + LK-G30) at the free end (unit in $\mathrm{mm}$ ): 1.7544, 2.4952, 2.9682, 3.3292, 3.5918, and 3.8152. The power analysis of SP-OSSHI and SEH circuits under different excitations is shown in Figure 13. It is easy to find that, under small excitation conditions, the power of the SP-OSSHI circuit is not significantly improved compared to that of the SEH circuit. It is mainly because the loss of the components in the SP-OSSHI circuit itself is greater than that in the SEH circuit. As the excitation becomes larger, the proportion of the loss of the circuit element itself to the extracted power gradually decreases, and the advantages of the SP-OSSHI circuit gradually appear. In the process of changing the peak-to-peak excitation from $1.7544 \mathrm{~mm}$ to $3.8152 \mathrm{~mm}$, the optimal power ratio of the SP-OSSHI circuit to the SEH circuit also increased from 1.24 to 1.95 . We can conclude that, with the increase of excitation, the energyharvesting potential of SP-OSSHI will gradually show up. Under higher excitation conditions, the energy-harvesting efficiency of the SP-OSSHI circuit will be much higher than that of the SEH circuit.

\subsection{Communication Verification of Self-Powered Wireless} Sensor Nodes. To match the energy extraction circuit and the wireless sensor node communication module, a self-powered wireless sensor node communication network is set up, as shown in Figure 14. Using the above SP-OSSHI circuit, the load is replaced with the power management module (Linear Technology; LTC3588). Storage capacitor $C_{\text {st }}$ is $2000 \mu \mathrm{F}$. The module can provide a stable and adjustable DC voltage supply for wireless sensor nodes. The power management circuit is shown in Figure 15. 


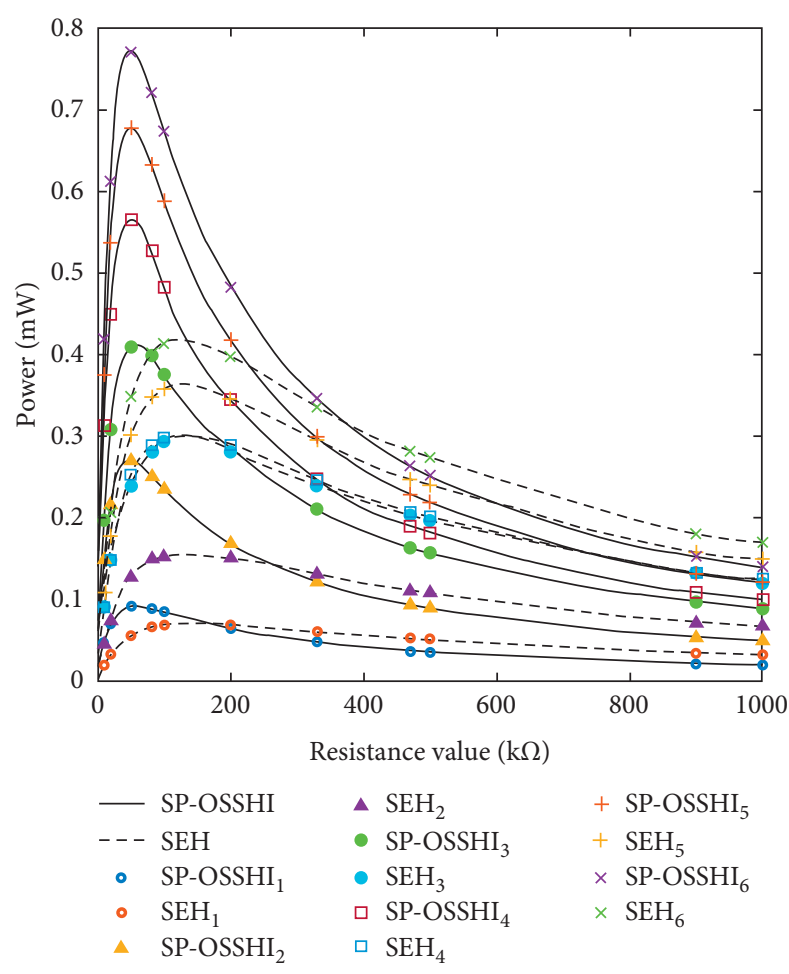

FIGURE 13: Various excitation circuit experiment waveforms.

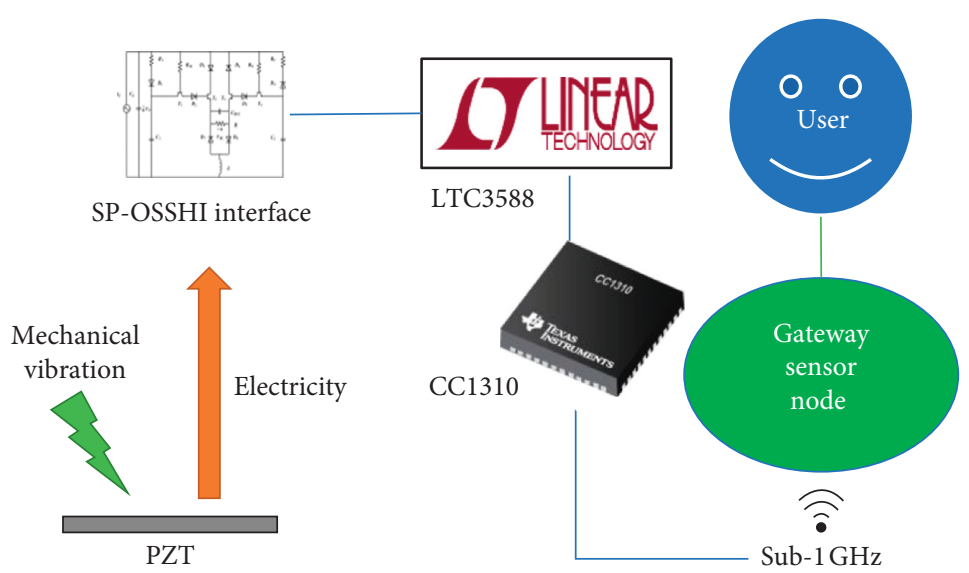

FIgURE 14: Self-powered WSN node.

In order to balance the energy harvesting and consumption, the power supply voltage is set to $3.3 \mathrm{~V}$, which is fit for the triaxial low-power accelerometer (Bosch; BMA250) and communication module (Texas Instruments; CC1310). The CC1310 module-integrated temperature monitoring sensor collects the ambient temperature data. The temperature and acceleration information are wirelessly transmitted to the CC1310 receiver (gateway sensor node) through the transmitter of CC1310 to complete the wireless transmission of the sensor data signal. The acquired data can be processed in real time on the host computer.

In this experiment, IIC communication is used. The process of sending and receiving bytes is shown in Figure 16.
The communication frequency is set to $433 \mathrm{MHz}$, and 32 bytes of data are sent through the transmitter. Taking the temperature data as an example, the data are transmitted to the CC1310 receiver, and the data are analyzed in the Code Composer Studio (CCS) integrated development environment (IDE) of the host computer software, and the data format obtained by the software decoding is shown in Figure 17. This wireless sensor node is totally self-powered with an interval of 30 seconds. After receiving the data, the receiver directly outputs the data to the host PC through the serial port. The power consumed during data acquisition and wireless sensing is shown in Figure 18.

The power loss during wireless transmission is $45 \mathrm{~mW}$, and the working time is $0.4 \mathrm{~s}$. The wireless transmission 


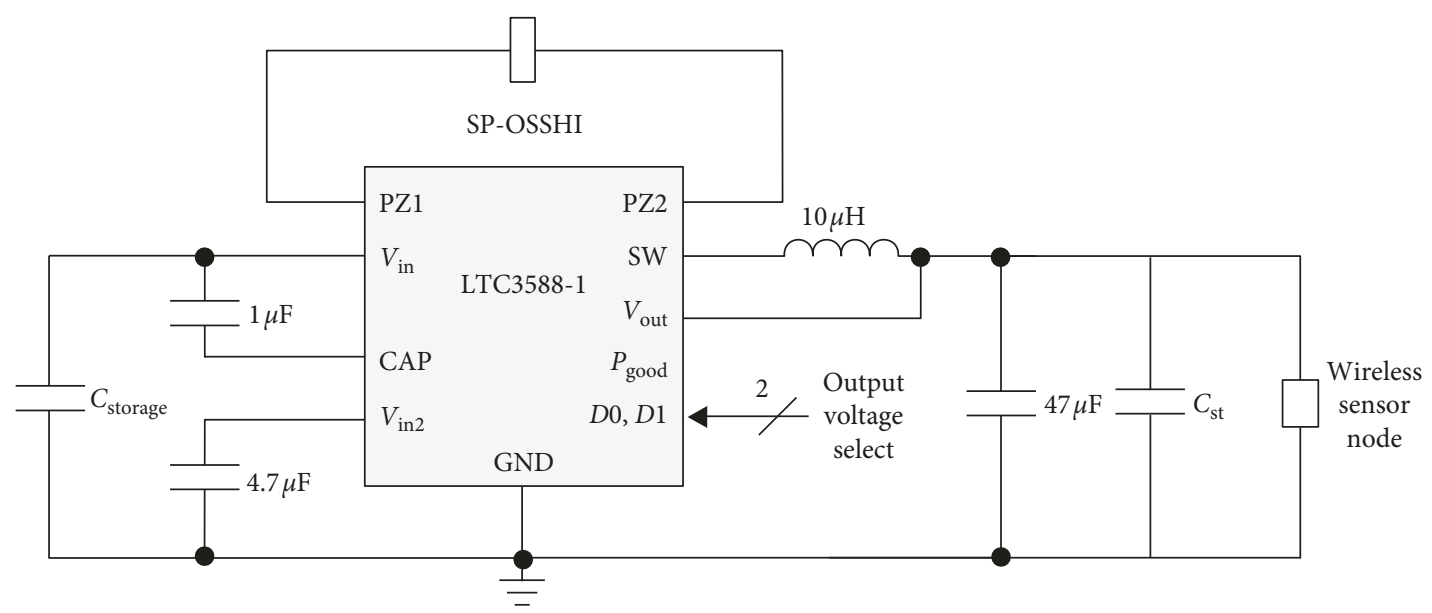

FIgURE 15: Power management circuit.

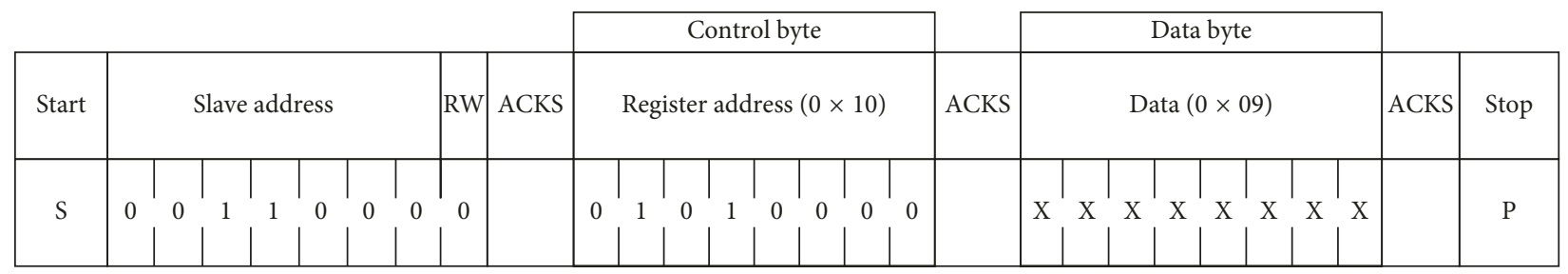

FIgURE 16: IIC protocol transmission flow chart.

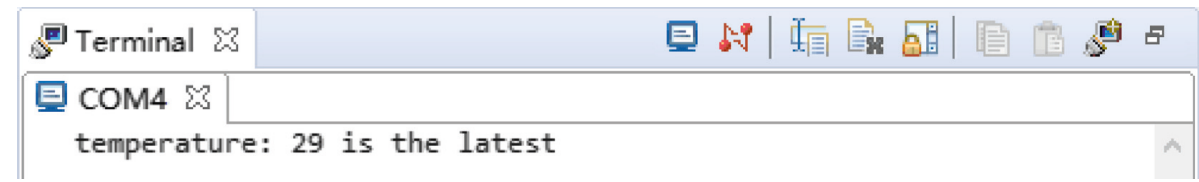

Figure 17: Temperature data format.

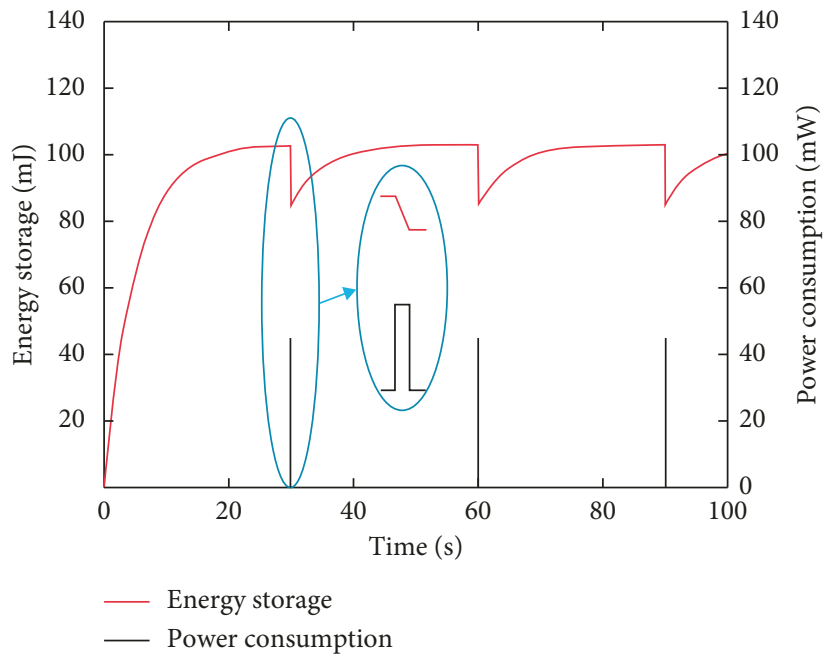

FIGURE 18: Energy consumption diagram of the transmission process. 
period is set to $30 \mathrm{~s}$, and the energy verified by the test can meet the transmission requirements.

\section{Conclusion}

In order to solve the power supply problem of wireless sensor node communication, a self-powered optimized synchronized switch harvesting on inductor (SP-OSSHI) circuit is used to harvest the vibration energy. Comparing and analyzing the power of the SP-OSSHI circuit with the power of the representative piezoelectric energy-harvesting $(\mathrm{PEH})$ circuit under different load resistance values, both the simulation result and the physical experiment prove that the energy-harvesting efficiency of the self-powered circuit is high and it is available to be used in industrial application. LTC3588 is used as a power management module and a supercapacitor as an energy storage unit to provide a stable power supply, with unlimited electricity income and power supply lifespan. The prototype including transmission modules CC1310 and BMA250 can be further modified according to different application scenarios. In the future study, we will focus on coupling the interface circuit with the load adjustment to further unlock the potential of self-powered energy-harvesting technique. A development board integrated with charge extraction circuit, power management, and transmission module will be designed as well.

\section{Data Availability}

The data (parameters and properties and model type) used to support the findings of this study are included within the article.

\section{Conflicts of Interest}

The authors declare that they have no conflicts of interest.

\section{Acknowledgments}

Grateful acknowledgement is given to the financial support from the National Natural Science Foundation of China (Grant No. 51805298), International Science \& Technology Cooperation and Exchang Program of Shaanxi Province, China (No. 2016KW-035), Fundamental Research Funds for the Central University (Chang'an University; No. 310832171007), and China Postdoctoral Science Foundation (No. 2015M580587).

\section{References}

[1] B. Zhang, D. Li, Y. Li et al., "Double peak derived from piezoelectric coefficient nonlinearity and proposal for selfpowered systems," Transactions of Nanjing University of Aeronautics and Astronautics, vol. 35, no. 1, pp. 109-115, 2018.

[2] S. Zhou and L. Zuo, "Nonlinear dynamic analysis of asymmetric tristable energy harvesters for enhanced energy harvesting," Communications in Nonlinear Science and Numerical Simulation, vol. 61, pp. 271-284, 2018.
[3] S. Sarkar and S. Misra, "From micro to nano: the evolution of wireless sensor-based health care," IEEE Pulse, vol. 7, no. 1, pp. 21-25, 2016.

[4] M. Winkler, K. D Tuchs, K. Hughes, and G. Barclay, "Theoretical and practical aspects of military wireless sensor networks," Journal of Telecommunications and Information Technology, vol. 2, no. 2, pp. 37-45, 2008.

[5] M. P. Zheng, Y. D. Hou, M. K. Zhu et al., "Research progress of piezoelectric ceramics for energy harvesting," Journal of the Chinese Ceramic Society, vol. 44, no. 3, pp. 359-366, 2016.

[6] D Pan, Y Li, and F Dai, "The influence of lay-up design on the performance of bi-stable piezoelectric energy harvester," Composite Structures, vol. 161, pp. 227-236, 2017.

[7] H. J. Rad, B. Abolhassani, and M. Abdizadeh, "Lifetime optimization via network sectoring in cooperative wireless sensor networks," Wireless Sensor Network, vol. 2, no. 12, pp. 905-909, 2010.

[8] S. Zhou and J. Wang, "Dual serial vortex-induced energy harvesting system for enhanced energy harvesting," AIP Advances, vol. 8, no. 7, article 075221, 2018.

[9] C. Dagdeviren, B. D. Yang, Y. Su et al., "Conformal piezoelectric energy harvesting and storage from motions of the heart, lung, and diaphragm," Proceedings of the National Academy of Sciences of the United States of America, vol. 111, no. 5, pp. 1927-1932, 2014.

[10] Y. K. Tan and S. K. Panda, "Optimized wind energy harvesting system using resistance emulator and active rectifier for wireless sensor nodes," IEEE Transactions on Power Electronics, vol. 26, no. 1, pp. 38-50, 2010.

[11] J. Zhang, D. Cao, Y. Cui, C. Putson, C. Song, and P. Huan, "Investigation of the influence of electron avalanche on the crystallinity of backsheet in solar photovoltaic system for sustainable energy," Journal of Cleaner Production, vol. 189, pp. 169-175, 2018.

[12] W. G. Li, S. He, and S. Yu, "Improving power density of a cantilever piezoelectric power harvester through a curved L-shaped proof mass," IEEE Transactions on Industrial Electronics, vol. 57, no. 3, pp. 868-876, 2010.

[13] P. Glynne-Jones, M. J. Tudor, S. P. Beeby, and N. M. White, "An electromagnetic, vibration-powered generator for intelligent sensor systems," Sensors and Actuators A: Physical, vol. 110, no. 1-3, pp. 344-349, 2004.

[14] P. D. Mitcheson, P. Miao, B. H. Stark, E. M. Yeatman, A. S. Holmes, and T. C. Green, "MEMS electrostatic micropower generator for low frequency operation," Sensors \& Actuators A Physical, vol. 115, no. 2-3, pp. 523-529, 2004.

[15] X. Wang, S. Niu, F. Yi et al., "Harvesting ambient vibration energy over a wide frequency range for self-powered electronics," ACS Nano, vol. 11, no. 2, pp. 1728-1735, 2017.

[16] D. Guyomar, A. Badel, E. Lefeuvre, and C. Richard, “Toward energy harvesting using active materials and conversion improvement by nonlinear processing," IEEE Transactions on Ultrasonics, Ferroelectrics, and Frequency Control, vol. 52, no. 4, pp. 584-595, 2005.

[17] E. Lefeuvre, A. Badel, C. Richard, and D. Guyomar, "Energy harvesting using piezoelectric materials: case of random vibrations," Journal of Electroceramics, vol. 19, no. 4, pp. 349-355, 2007.

[18] E. Lefeuvre, A. Badel, C. Richard, and D. Guyomar, "Piezoelectric energy harvesting device optimization by synchronous electric charge extraction," Journal of Intelligent Material Systems and Structures, vol. 16, no. 10, pp. 865-876, 2005.

[19] M. Lallart, L. Garbuio, L. Petit, C. Richard, and D. Guyomar, "Double synchronized switch harvesting (DSSH): a new 
energy harvesting scheme for efficient energy extraction," IEEE Transactions on Ultrasonics Ferroelectrics \& Frequency Control, vol. 55, no. 10, pp. 2119-2130, 2008.

[20] M. Lallart, "Nonlinear technique and self-powered circuit for efficient piezoelectric energy harvesting under unloaded cases," Energy Conversion and Management, vol. 133, pp. 444-457, 2017.

[21] Y. Wu, A. Badel, F. Formosa et al., "Piezoelectric vibration energy harvesting by optimized synchronous electric charge extraction," Journal of Intelligent Material Systems and Structures, vol. 24, no. 12, pp. 1445-1458, 2013.

[22] B. Zhang, B. Ducharne, D. Guyomar, and G. Sebald, "Energy harvesting based on piezoelectric Ericsson cycles in a piezoceramic material," European Physical Journal Special Topics, vol. 222, no. 7, pp. 1733-1743, 2013.

[23] B. Zhang, B. Ducharne, B. Gupta, G. Sebald, D. Guyomar, and J. Gao, "Experimental sea wave energy extractor based on piezoelectric Ericsson cycles," Journal of Intelligent Material Systems and Structures, vol. 29, no. 6, pp. 1102-1112, 2018.

[24] E. Lefeuvre, A. Badel, A. Brenes, S. Seok, and C.-S. Yoo, "Power and frequency bandwidth improvement of piezoelectric energy harvesting devices using phase-shifted synchronous electric charge extraction interface circuit," Journal of Intelligent Material Systems and Structures, vol. 28, no. 20, pp. 2988-2995, 2017.

[25] S. Bagheri, N. Wu, and S. Filizadeh, "Modeling of capacitor charging dynamics in an energy harvesting system considering accurate electromechanical coupling effects," Smart Materials and Structures, vol. 27, no. 6, article 065026, 2018.

[26] K. Tao, L. Tang, J. Wu, S. W. Lye, H. Chang, and J. Miao, "Investigation of multimodal electret-based MEMS energy harvester with impact-induced nonlinearity," Journal of Microelectromechanical Systems, vol. 27, no. 2, pp. 276-288, 2018.

[27] Z. Zhang, H. Xiang, Z. Shi, and J. Zhan, "Experimental investigation on piezoelectric energy harvesting from vehiclebridge coupling vibration," Energy Conversion and Management, vol. 163, pp. 169-179, 2018.

[28] D. Su, Y. Xia, and R. Yuan, "Self-powered wireless sensor network for automated corrosion prediction of steel reinforcement," Journal of Sensors, vol. 2018, Article ID 4125752 , 10 pages, 2018.

[29] Y. Liao and J. Liang, "Maximum power, optimal load, and impedance analysis of piezoelectric vibration energy harvesters," Smart Materials and Structures, vol. 27, no. 7, article 075053, 2018.

[30] A. A. Babayo, M. H. Anisi, and I. Ali, "A review on energy management schemes in energy harvesting wireless sensor networks," Renewable and Sustainable Energy Reviews, vol. 76, pp. 1176-1184, 2017.

[31] M. Lallart and D. Guyomar, "An optimized self-powered switching circuit for non-linear energy harvesting with low voltage output," Smart Materials and Structures, vol. 17, no. 3, article 035030, 2008.

[32] C. Richard, D. Guyomar, and E. Lefeuvre, "Self-powered electronic breaker with automatic switching by detecting maxima or minima of potential difference between its power electrodes," Patent PCT/FR2005/003000, 2007, (publication number: WO/2007/063194).

[33] J. Liang and W. H. Liao, "Improved design and analysis of self-powered synchronized switch interface circuit for piezoelectric energy harvesting systems," IEEE Transactions on Industrial Electronics, vol. 59, no. 4, pp. 1950-1960, 2012. 


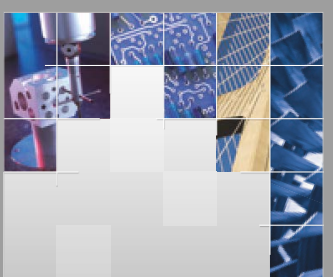

\section{Enfincering}
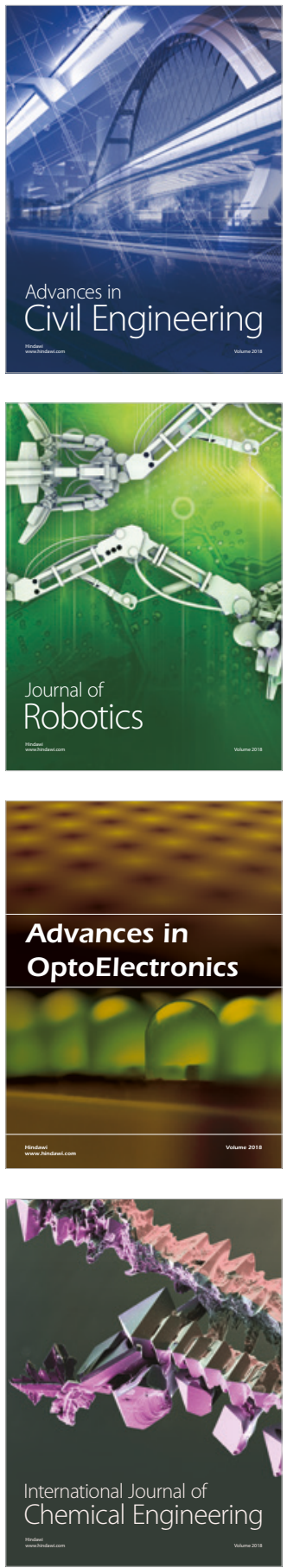

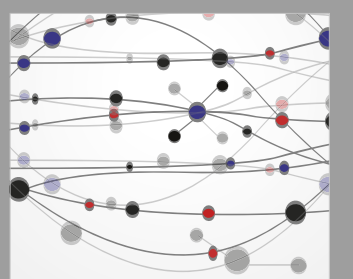

\section{Rotating \\ Machinery}

The Scientific World Journal

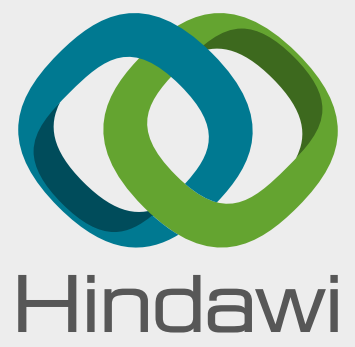

Submit your manuscripts at

www.hindawi.com
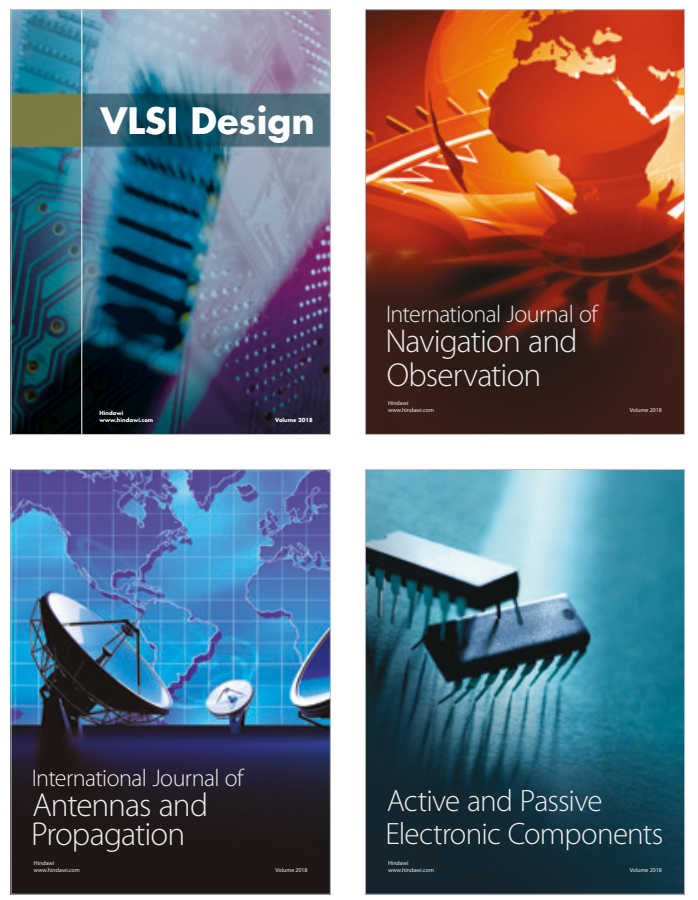
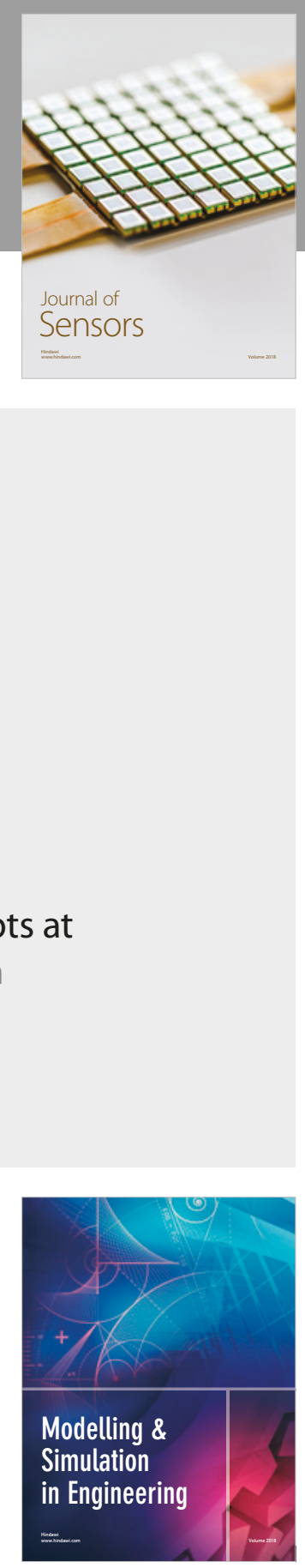

\section{Advances \\ Multimedia}
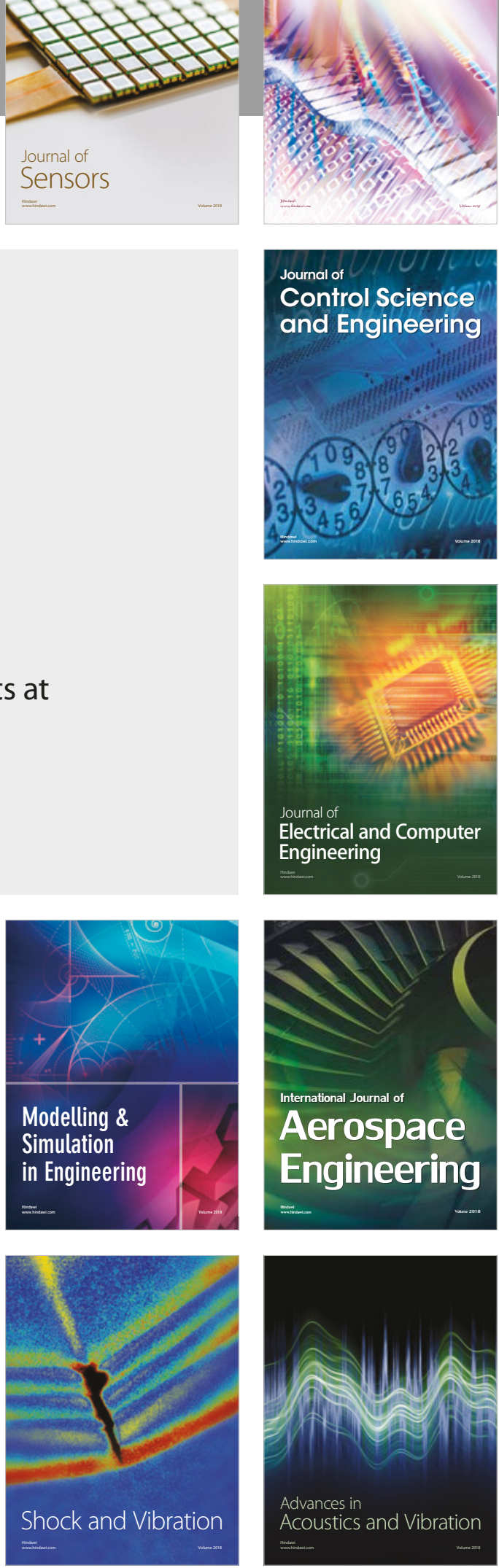